\title{
Photoelastic study of dense granular free-surface flows
}

\author{
A. L. Thomas* and N. M. Vriend \\ Department of Applied Mathematics and Theoretical Physics, University of Cambridge, Wilberforce Rd, Cambridge CB3 OWA, \\ United Kingdom
}

(Received 23 October 2018; revised manuscript received 22 April 2019; published 8 July 2019)

\begin{abstract}
In this study, we perform experiments that reveal the distribution of dynamic forces in the bulk of granular free-surface flows. We release photoelastic disks from an incline to create steady two-dimensional avalanches. These gravity-driven dry granular flows are in the slow to intermediate regime $(I \leqslant 1)$, dense $(\varphi \approx 0.8)$, and thin $(h \approx 10 d)$. The transition between solidlike (quasisteady) and fluidlike (inertial) regimes is observable for certain experimental settings. We measure constant density and quasilinear velocity profiles through particle tracking at several points down the chute, for two different basal topographies. The photoelastic technique allows the visualization and quantification of instantaneous forces transmitted between particles during individual collisions. From the measured forces we obtain coarse-grained profiles of all stress tensor components at various positions along the chute. The discreteness of the system leads to highly fluctuating individual force chains which form preferentially in the directions of the bulk external forces: in this case, gravity and shear. The behavior of the coarse-grained stress tensor within a dynamic granular flow is analogous to that of a continuous fluid flow, in that we observe a hydrostatic increase of the mean pressure with depth. Furthermore, we identify a preferential direction for the principal stress orientation, which depends on the local magnitudes of the frictional and gravitational forces. These results allow us to draw an analogy between discrete and continuous flow models.
\end{abstract}

DOI: 10.1103/PhysRevE.100.012902

\section{INTRODUCTION}

Granular materials can display behaviors reminiscent of solids, liquids, or even gases depending on the mean particle energy. Analogies with such systems have helped characterize different granular regimes, but particularly intermediateenergy systems often present phenomena that are unique to granular structures $[1,2]$. In a granular regime, the marked differences with typical fluids are attributed to the existence of static friction, the fact that temperature does not play a role, and the inelastic nature of collisions [3]. Until the roles of these differences are fully understood, it will be difficult to develop a unified theory for the rheology of granular flows. The combined complexities of interparticle interactions result in the inhomogeneous distribution of stresses through force chains [4]. We consider chains to be the result of the combined effect of the properties that make granular systems unique and that are difficult to evaluate individually. This work therefore focuses on the characterization of force chains in dynamic avalanches.

Due to the large variability and complexity of the force network formation, much work on its characterization has been approached through statistical modeling. The probability density of contact forces has been identified as a key quantitative feature in the understanding of the inhomogeneous nature of stress transmission in granular media, particularly in the study of jamming and yielding [4-7]. However, a full understanding of the network and the effect of its topology also requires information on the force chain's spatial arrangement, arching,

\footnotetext{
*at682@cam.ac.uk
}

and branching [8]. Snoeijer et al. [9] introduced a method to combine this theory with a model for the ensemble of possible network arrangements for a given fixed arrangement of particles. Notwithstanding, the modeling of granular force networks has proven complex even for static configurations of particles. More so, the characterization of force networks in dynamic systems is to this day markedly underrepresented.

An important factor in the slow progress in the theoretical modeling of granular force networks is the fact that models are difficult to validate experimentally. Most rheological models for granular free-surface flows are therefore based on numerical simulations [10-13], where the forces exerted between particles can be estimated through the contact model of choice. Although these studies have indeed supplied invaluable insight, the limitation of results obtained through numerical simulations is that they are susceptible to carrying the errors of the theoretical models they are based on. It is essential that real-life experiments validate how realistic numerical results are. Experimental studies do exist [14-17] where the kinematic flow properties are inferred from particle tracking through chute side walls. However, because the link between the flow kinematics and dynamics is not yet fully understood, limited experimental information on the distribution of forces within granular flows has been obtained to this date.

In the following sections, we describe an original and innovative experiment where we apply the photoelastic technique to quantify all the individual forces exerted during interparticle collisions within avalanches. Photoelasticity has been the stepping stone onto many of the advances made in our understanding of, for example, jamming [18-21], slope failure [22], and force propagation [23-25]. Still, for large systems the computational cost of resolving forces remains 
time consuming and expensive because high spatial and temporal resolution is required to image many disks with enough detail. Other experimental methods to quantify forces based on the particle positions and deformations have been attempted [26-28], but the photoelastic technique remains the most accessible. However, as far as the authors of this paper are aware, none have yet quantified forces in dynamic systems where torque and particle velocity play a significant role.

The novelty of this work lies in the experimental, rather than numerical or theoretical, measurement of forces within the bulk of dynamic two-dimensional (2D) free-surface granular flows. We first explain the principles behind the measurement of forces from photoelastic patterns. The application of the photoelastic technique in a dynamic system where torque and momentum are not balanced is unique, and here we briefly discuss the adaptations made to the classical approach. Next, we characterize the state of the flow by presenting the coarse-grained packing fraction and velocity profiles obtained through particle tracking. We then use the discrete interparticle forces measured experimentally to analyze the preferential direction and strength of the average force chain. These discrete experimental results are also coarse grained to obtain continuous profiles for the internal stress tensor components. The dense, slow, and thin regime explored in this work allows the rheological study of the transition between solidlike and fluidlike behavior of granular systems through the formation of a superstable heap ( $\mathrm{SSH}$ ). Throughout the Results section, we compare the observations made to other reports of both $2 \mathrm{D}$ and three-dimensional (3D) flows confined within a narrow channel, while simultaneously pioneering in the experimental observation of the force-chain network. Finally, we report the conclusions drawn from our experimental results, and explore the extent to which granular flows are analogous to classic fluid dynamics.

\section{EXPERIMENTS}

\section{A. Experimental technique}

The experimental technique applied in this study is based on the material property of photoelasticity, by which certain materials become birefringent in a degree proportional to the magnitude of external loads [29]. In other words, the refractive index of these materials at each point depends on the internal stress magnitudes. As a result, the material may have different refractive indices in the different principal stress directions. If this is the case, the light that travels through the material will experience a change in its polarization, enabling direct observation of forces in photoelastic systems.

Consider a setup of two opposite circular polarizing films in parallel, positioned as shown in Fig. 1. As they are opposite in polarization directions, the pair will absorb all the light that passes through them. If a birefringent sample material is placed in-between them, circularly polarized light transmitted by one of the polarizers will no longer be circular after it is transmitted through the sample. This is because the polarization of the transmitted light is affected by the sample having different directional refractive indices. As a result, the light transmitted through the second polarizer will no longer be circularly polarized, but will have a residual phase change

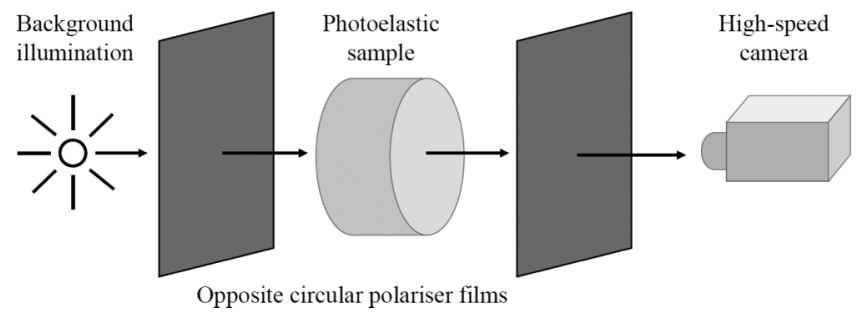

FIG. 1. Sketch of the basic setup needed to visualize the photoelastic response of a sample.

upon leaving the sample. On reaching the second polarizer, the component of this light corresponding to a circular polarization is absorbed by the second polarizer, while the component associated to the change of phase may be transmitted through the setup. The intensity of the light transmitted through this system depends on the degree of phase shift created by the sample, which depends on the internal stress magnitudes, which in turn can be related to the external forces applied on the photoelastic sample.

An outside observer sees a black background with bright light patterns visible inside the photoelastic material, an example of which is shown in Fig. 2(a). These patterns depend on the magnitude and direction of the loads applied to the material. By measuring the pixel-by-pixel intensity of the light transmitted in this system, we deduce the internal stresses and from them the magnitudes of the external forces acting on the sample. The quantitative relationship between the observed light intensity and the external forces is not linear nor straightforward [30,31]. For a reliable measurement, the forces must instead be calculated by postprocessing the photoelastic light patterns, or fringes. If the distribution of internal stresses of a specific photoelastic sample can be modeled, then the fringe pattern can be predicted and compared to the experimental observation. Figure 2(b) shows the photoelastic fringe pattern expected given a specific pair of forces acting on a disk. Via an iterative optimization algorithm, the forces that would produce a specific fringe pattern can be converged into with very high precision (with errors as low as 5\% [32,33]). In Fig. 2 the patterns observed in the experimental image and the theoretical reconstruction look very similar because the force magnitudes used to produce Fig. 2(b) are indeed the values measured when compressing the disk in Fig. 2(a).

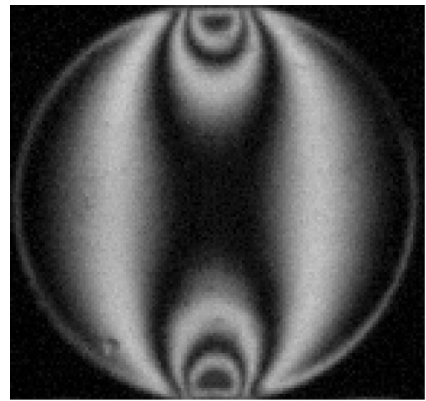

(a)

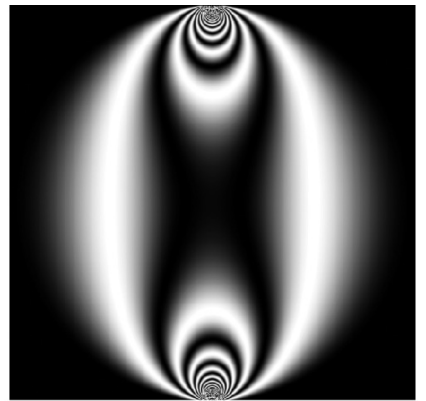

(b)
FIG. 2. (a) Example of a photoelastic disk under two opposite concentric known forces, and (b) theoretical fringe pattern [30,34] produced for the experimental forces. 


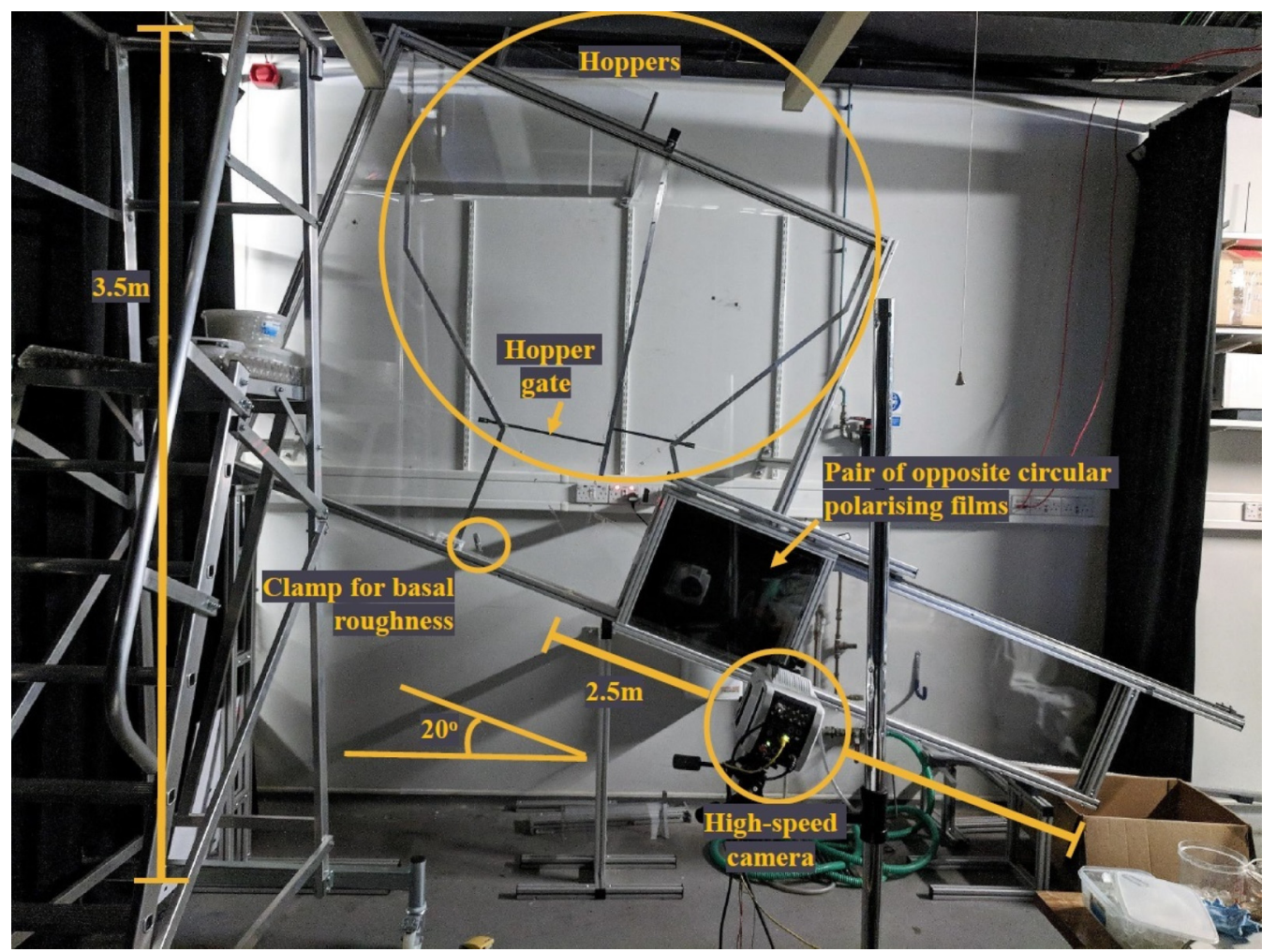

FIG. 3. Experimental setup for the observation of forces within a 2D avalanche of photoelastic disks.

For this work in particular, Clear Flex 50 (Smooth-on) was used to cast the photoelastic disks mimicking a procedure developed by Barés [35,36]. Although less photoelastically responsive than other popular materials (see Fig. 8 in Daniels and Puckett [32] for the calibration plot), Clear Flex 50 was chosen because it can be cast into any shape with no residual stresses and the cured product shows minimal light diffusion. As a result, the experimental images observed are remarkably similar to the theoretical construction. The main difference in the patterns observed experimentally and those produced theoretically [34] is around the force application point. The model assumes the force is applied at a single point, which is unrealistic because in practice pressures are applied over a small area that deforms slightly.

The approach we apply to estimate forces from photoelastic patterns by solving the optimization problem is often referred to as the inverse method. As we apply it, the limits of the technique lie in the temporal and spatial resolution of the experimental images (due to camera resolution and diffusion in the photoelastic material) and in the tradeoff between computational cost and accuracy. High-resolution imaging of each photoelastic disk is needed to measure forces accurately, and the dynamic nature of our system requires our capture frame rate to be much higher than the frequency of network force rearrangement. Moreover, the constraint introduced by force equilibrium in static arrangements is released in the case of flowing particles, increasing the computational cost of the inverse method calculations for a given target accuracy. These technical challenges have been the reason why previous studies preferred to use the photoelastic technique to study static arrangements only, and to use estimative methods, such as the $G^{2}$ method [37-40], instead of the inverse method, to resolve forces from experimental images. Recent advances in high-speed imaging and computational resources have now allowed us to overcome such experimental challenges to study dynamic flow configurations.

\section{B. Experimental setup and procedure}

The experiment is set up as shown in Fig. 3, so as to observe a single layer of 6-mm-thick photoelastic disks avalanche down an incline. Two large acrylic panels are pressed together and separated by $8 \mathrm{~mm}$ of plastic and aluminum framework, providing enough space to allow the disks to roll freely with minimal resistance. The complete setup is just over $3 \mathrm{~m}$ high and $3.5 \mathrm{~m}$ long, and permanently inclined at an angle of $20^{\circ}$ to the floor.

Before an experiment, Clear Flex 50 disks of 11, 12, and $13 \mathrm{~mm}$ in diameter (all $6 \mathrm{~mm}$ in thickness) were introduced in equal numbers from above into either or both hoppers seen on the top left of the setup. The approximate $10 \%$ polydispersity causes a randomness in disk size distribution large enough to prevent crystallization [41], but small enough that segregation is negligible. Indeed, neither phenomenon is observed in the flows discussed henceforth. To reduce the frictional effect of the chute walls on the flowing disks, they are regularly lightly coated in flour.

We then manually slide a plastic gate located at the base of each hopper (visible as dark gray strips in Fig. 3) to release the disks at a constant flow rate. The particles fall into a channel, formed by two 2-m-long and 0.5 -m-high acrylic sheets which 


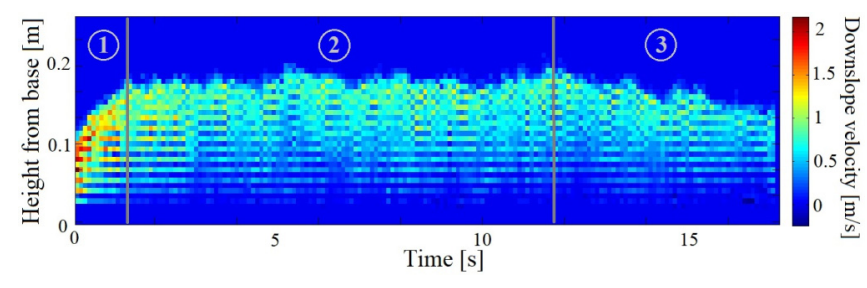

FIG. 4. Evolution in time of the binned particle streamwise velocities as they pass through a mark $75 \mathrm{~cm}$ downstream from the hopper.

are also clamped $8 \mathrm{~mm}$ apart. A plastic strip with glued semidisks can be fixed to the base of the inclined chute to introduce basal friction. Half of the experiments were carried out without any basal roughness (smooth base case), while the other half were performed over a layer of semidisks (of the same diameters, $12 \mathrm{~mm}$, as the flowing disks average) glued to the strip clamped to the channel base (rough base case).

Simultaneously to the release of the gate, we trigger a Phantom v2012 Ultrahigh-Speed Camera (Vision Research) to record the flow of photoelastic disks at a fixed position down the avalanche channel. By progressively increasing the recording frame rate, we determined that the loading and unloading at the contact between particles can take as little as $1 \mathrm{~ms}$. Hence, we set the camera exposure to $1 / 8000 \mathrm{~s}$, corresponding to the highest frame rate at which the range of light intensities recorded was not compromised, but we save only 1000 frames per second to allow for rearrangements between captures.

The background illumination is provided by a TruOpto OSPM-R5030ETS Red LED, while a pair of opposite circular polarizers, one on either side of the chute, reveals the disks' photoelastic response to forces exerted on them. We can adjust the target location for measurements along the chute by moving the combination of LED lights and polarizers along a runway on top of the chute, after which the external camera field of view can be aligned. When the Clear Flex 50 disks roll in-between the polarizers, the camera captures the photoelastic response to interparticle interactions as bright light patterns on a dark background.

Figure 4 shows the velocity profile time series measured at a location $75 \mathrm{~cm}$ from the hopper for an experiment performed over a rough base. The experimental videos of the flow past a given point are divided into three stages: (1) initially the flow quickly thickens and slows as the avalanche head passes the observation point, until it reaches (2) a steady state where a constant height and velocity profile is maintained for several seconds, after which (3) the flow decelerates and thins. For this work, we ignore the transient developments and focus on the middle section in the time series instead, marked (2) in Fig. 4, which is characterized by a constant flow height. From this steady-state stage, we collect up to 3000 frames in each experiment, but as postprocessing is such a time-consuming and expensive process, only 500 frames (equivalent to $0.5 \mathrm{~s}$ of flow) were used to analyze photoelastic patterns. We tested with different sets of 500 frames within this steady-state region and always observed the same averaged profiles, so we determined that 500 frames is a large enough frame sample number to obtain results representative of the whole stage.

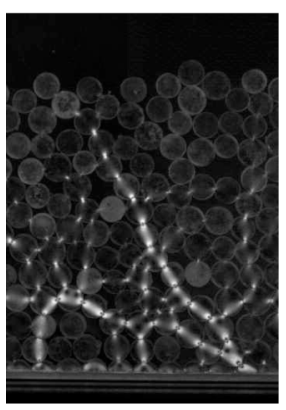

(a)

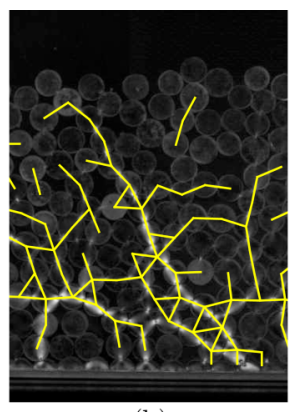

(b)

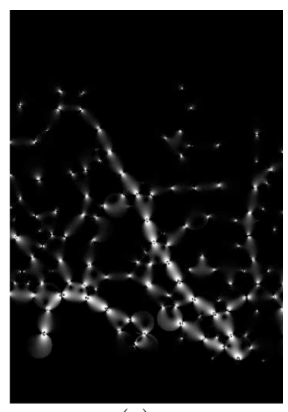

(c)
FIG. 5. (a) Example experimental frame; (b) identification of significant forces, measured using PEGS, that form the force-chain network; and (c) reproduced photoelastic patterns predicted from the measured forces.

Finally, a large box placed beneath the lower end of the incline collects the disks as they exit the lower end of the chute freely. In the rough base cases, a layer of static disks, forming an angle in the laboratory frame of about $23^{\circ}$, remains deposited on the base even after the inflow stopped.

\section{Experimental data extraction \\ 1. Discrete particle data}

Figure 5(a) shows a typical experimental image as captured by the camera. The outlines of the disks are visible due to minor light pollution from the surrounding; something that is allowed on purpose to aid the identification of particle position. We apply the MATLABfunction imfindcircles, with empirically tested parameters, in postprocessing to every experimental image to locate all disks in the middle half of the image (where the background lighting is consistent). As the frame rate is so high, the circles identified move at most only a few pixels between frames, so they are easily tracked throughout the experiments. Depending on the specific particle edge visibility and the instantaneous photoelastic pattern brightness, we estimate a location error of at most $1 \mathrm{~mm}$, or $\approx 10 \%$ of the mean diameter. Some disks appear slightly murky due to the random settling of the flour coating on their surface. As the photoelastic fringes are considerably brighter, the error introduced by this noise is considered negligible.

The flow depicted in Fig. 5(a) is visibly very dense, with all disks being in contact with most of their neighbors at any time. We determine from the data collected during the particle tracking that the coordination number of the system is $4.2 \pm$ 0.3 , which is consistent throughout all experiments, over both smooth and rough bases. Nevertheless, Fig. 5 illustrates the property of granular systems by which not all contacts carry the same loads, which results in only a small proportion of the total particles taking part in the force network. The wide black gaps between force chains are filled with particles that do not exhibit an obvious instantaneous photoelastic response to any force. The proportion of disks involved in the force network increases with depth, but in the experimental frames analyzed no more than $30 \%$ of disks form part of force chains.

The force-measuring code was built on the open-source program PEGS written by Kollmer [32,33]. This program extracts the light intensity distribution inside the circles located 
by the imfindcircles function. We assume two disks are in contact when their circumferences are closer than the disk location error. As we are only interested in load-bearing contacts, we discard those whose $G^{2}$ values (sum of the pixel intensity gradient squared) near the contact do not exceed a minimum value determined empirically. PEGS then uses the $G^{2}$ method [37-40] to estimate an initial guess for the optimization algorithm that solves the inverse problem. This particular code was used because it offers the option to enable or disable the condition of equilibrium (force and torque balance) at the user's discretion. Most existing work quantify forces among static disks in equilibrium, but PEGS is able to analyze dynamical systems that lack force equilibrium. If applicable, this condition is used to simplify the internal stress distribution model and to provide a quicker initial guess for the iterative optimization algorithm. Both these parts of the code were adapted for its application to dynamic disks in an avalanche, at the cost of higher computational processing times.

The experimental technique limitations depend on the inherent photoelastic response of Clear Flex 50, the thickness of the disks, and the image resolution intrinsic to the camera. In our current setup we are limited by the sensitivity lower bound of $0.02 \mathrm{~N}$, and a systematic error of $0.05 \mathrm{~N}$ exists for forces of magnitudes smaller than $0.5 \mathrm{~N}$. On the other hand, the random error of the technique can be as large as $20 \%$ between this lower bound and a critical value that depends on the number of forces acting on the disk $z$. The reliability of the force measurements drops significantly when the loads on the disks exceed $2.5 \mathrm{~N}$ when $z=2,1.6 \mathrm{~N}$ when $z=3$, and $1.2 \mathrm{~N}$ when $z=4$. In our experiments, approximately 1 in 100 disks is subject to three or four force-bearing contacts, and in these cases two of them are usually dominant by at least an order of magnitude. See the Appendix for the details of how these values were obtained.

Given that the average disk mass is $m_{i} \approx 0.78 \pm 0.04 \mathrm{~g}$, only forces that are at least three times greater than the average disk weight will produce a visible photoelastic response, which indeed rules out a great majority of the contacts as bearers of significant loads. On the other hand, disks that are involved in force chains carry loads that are on average at least an order of magnitude larger than the minimum. It is important to be aware that a large number of small forces are neglected in the following analysis, as the technique sensitivity naturally filters the force chains. The experiments still provide much valuable information about the structure and general statistics of the force network. Figure 5(b) shows yellow (light gray) thick solid lines along the network found for a particular example frame.

The overall experimental result of running the selected frames through the photoelastic force-calculating program, PEGS [32], is a list per frame of tracked disks, each of which has associated to it a unique $i d$ number, its radius, center coordinates, disk velocities, and a list of force vectors acting on the disk. Each force measured is recorded as a structure including the magnitude, direction, point of application, and the id of the disk that applies each load. Figure 5(c) shows the photoelastic pattern reproduced in postprocessing using the contact locations and forces measured from experimental images. The qualitative and quantitative similarity of
Fig. 5(c) with the original experimental image (a) endorses the technique's reliability.

\section{Continuous flow data}

To obtain continuous profiles from the list of discrete experimental results, we applied the coarse-graining approach described by Goldhirsch [42] and Weinhart et al. [43]. We apply the same coarse-graining equations for density, velocity, and stress components proposed by these groups, but in this work the coarse-graining function used was

$$
\varphi(\mathbf{r}, w)= \begin{cases}C e^{\frac{1}{1-\left|\left(\mathbf{r}-\mathbf{r}_{i}\right) / w\right|^{2}}}, & \left|\mathbf{r}-\mathbf{r}_{i}\right|<w \\ 0, & \left|\mathbf{r}-\mathbf{r}_{i}\right| \geqslant w\end{cases}
$$

where $\varphi$ represents the coarse-graining function that, at point with coordinates $\mathbf{r}$, depends on the distance to the center of each disk, $\mathbf{r}_{i}$, in terms of a predefined coarse-graining length scale $w$, which may have different components in the vertical and horizontal directions $\mathbf{w}_{\mathbf{z}}$ and $\mathbf{w}_{\mathbf{x}}$, respectively. $C$ represents the total volume of $\varphi$ over the whole domain. The function $\varphi$ can be chosen arbitrarily, as long as it satisfies [44] that it is non-negative $\varphi \leqslant 0$ (ensuring the density $\rho$ is always positive, and that the moment $p$ has the same sign as the particle velocity); that it is spatially normalized $\int_{\mathbf{R}^{3}} \varphi=1$ (to hold mass and momentum conservation); and that there exists a cutoff $c$ such that $\varphi(\mathbf{r})=0$ for $|\mathbf{r}|>c$ (ensuring that the effect of each variable is constrained within a finite distance defined by the length scale $w$ ). Gaussian functions $[45,46]$ and Lucy polynomials $[43,44]$ are popular choices for $\varphi$, but we use Eq. (1) because aside from satisfying all necessary requirements, it naturally tends to zero at lengths equal to $\mathbf{w}$ and thus provides an easy visualization of the coarse-graining length scale based only on w. The function $\varphi$ spans over an ellipse of height and width equal to the vertical and horizontal coarse-graining lengths.

The ideal coarse-graining length scale $w$ depends on the system and on the coarse-graining function $\varphi$. In each case, Goldenberg et al. [47] stipulated the optimal $w$ is such that it is large enough to average over microscopic changes (for example, mass variations between individual grains) but small enough to not average over macroscopic changes (for example, if part of a system has lower packing fraction than another). In other words, $w$ must be the smallest value such that using the function $\varphi(w)$ results in smooth profiles within sub- $w$ length scales. For the experiments described in this paper, the optimal $w_{z}$ was found to be $3 d$. However, we notice that the flow does not change significantly in the streamwise direction (horizontal $\hat{x}$ ), while it does vary rapidly in the crossflow direction $(\hat{z})$. We therefore set $w_{x}=5 d$, which allows us to coarse grain over the largest possible area within the middle half of the images where the background intensity is uniform.

\section{FLOW CHARACTERIZATION}

\section{A. Density profiles}

In order to analyze the grain density distribution, we coarse grain $[42,43]$ the particle mass and position information obtained by particle tracking. We defined $w_{z}=3 d$ as the optimal coarse-graining length scale to obtain smooth profiles, averaged over microscopic variations. However, by using a 


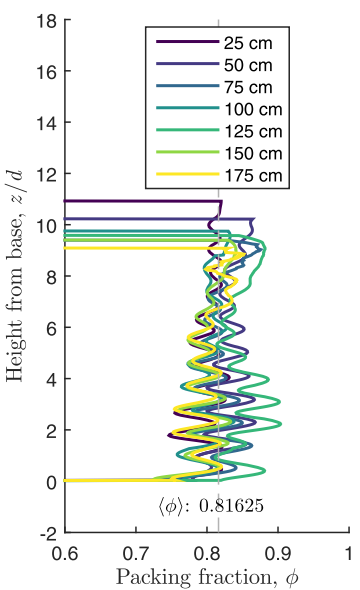

(a)

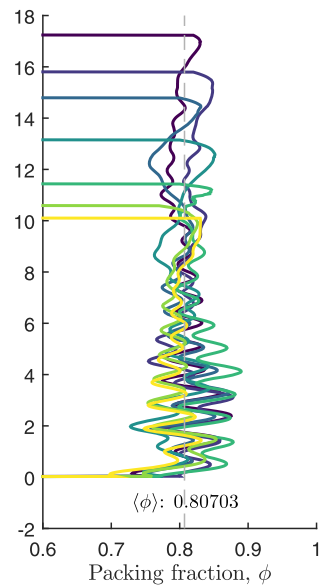

(b)
FIG. 6. Coarse-grained density profiles at seven positions down the chute for flows over (a) a smooth base and (b) a rough base. Both figures apply the same color scheme for the profiles measured at different downstream positions.

$w_{z}<3 d$ for this particular analysis only, we forfeit a smooth profile in lieu of a plot where we can observe the effect of layering in the packing fraction profile. For the following analysis, we half the optimal $w_{z}$, setting it to the arbitrarily small value of $1.5 d$, so that microscopic vertical changes can be observed, but we keep $w_{x}=5 d$ in order to ensure the coarse-graining space is large and representative of the flow.

The particle coordinates tracked using the MATLAB function imfindcircles were coarse grained according to the expression proposed by Goldhirsch [42] along the central vertical line on each frame. This line passes through the cross section of the chute at fixed downstream distances of exactly 25, 50, $75,100,125,150$, and $175 \mathrm{~cm}$ away from the hopper gate. A vector of coarse-grained densities $\rho_{\mathrm{CG}}(z)$, with a length equal to the vertical pixel resolution, can be obtained for different depths within the flow. This vector is produced for every frame and the results for 500 frames were averaged to obtain a single plot of density versus height from the chute base. Figure 6 shows a representation of these data converted into packing fraction (we measured cured Clear Flex 50 to have a density of $1120 \mathrm{~kg} / \mathrm{m}^{3}$ ) as a function of height for different downstream positions (shown in different colors, or shades of gray) and basal roughnesses [for Fig. 6(a) the smooth base and Fig. 6(b) the rough base].

Several interesting features of Fig. 6 stand out. First, the flow thickness is noticeably affected by the basal topography. The flow thickness across the smooth base remains fairly constant in downstream direction, but thins dramatically in the rough base by $60 \%$. Interestingly, the thickness at the bottom of the chute $(x=175 \mathrm{~cm})$ in both roughness cases is almost identical.

Second, the average packing fraction is effectively constant throughout the flow thickness at about $\phi_{\text {avg }}=0.81 \pm 0.07$ for all experiments performed. This result is only slightly smaller than the $2 \mathrm{D}$ random close packing fraction of $\phi_{\text {rcp }}=0.84$ for 2D systems made of monodispersed circles [48], bearing in mind that the experiments performed in this work have approximately $10 \%$ polydispersity. Aside from justifying the assumption of a uniform constant density, the similarity between $\phi_{\text {avg }}$ and $\phi_{\text {rcp }}$ unequivocally places all the experiments discussed into the same regime of dense granular flows.

The third relevant characteristic of Fig. 6 is that, despite the broad coarse-graining area, wide undulations can be seen at sub- $w_{z}$ distances. Although an even longer vertical coarse-graining length scale would provide a smoother profile, important physical meaning can be deduced from this behavior. We observe in Fig. 6 for all downstream positions that the undulations all show the same wavelength but their amplitude increase with depth. An autocorrelation analysis for both smooth- and rough-base experiments shows a consistent separation between density peaks of $1.08 \pm 0.02 \mathrm{~cm}$, which is equivalent to $0.9 d$, throughout the flow depth. This result implies that the disks flow in well defined layers, which are equally distanced throughout the depth, but with smaller vertical velocity fluctuations closer to the base. The fact that the undulations decrease in amplitude, but not in wavelength, with height, suggests a higher disorder at the top of the flow, where disks move at the fastest velocity. These results agree with the Weinhart et al. [49] numerical study of dry, frictional, steady-state granular flows down rough inclines, where they found that particles flowed in slightly interlocked layers separated by $0.907 \mathrm{~d}$. This was true for all slow and intermediate flows and they also observed that the layering was most organized closer to the base but decayed over larger distances for slower flows $(I \leqslant 0.2)$.

If we model mass to be distributed normally around $\mathbf{r}_{i}$, with variance $\sigma^{2}$, then the standard deviation $\sigma$ effectively represents the mean squared vertical displacement $\left\langle\delta z^{2}\right\rangle$ of the disks from the $i$ th layer midpoint. Knowing that layers contain the same particle density and that they are all equally spaced by $0.9 d$, we model the corresponding coarse-grained density profile, calculated as

$$
\rho_{\mathrm{CG}}^{n}(\mathbf{r})=\sum_{i=1}^{N} \varphi\left(\mathbf{r}-\mathbf{r}_{i}\right) \int_{-\infty}^{\infty} m_{i}\left(\mu_{i}, \sigma_{i}^{2}, z\right) d z,
$$

where the mass function $m_{i}$ for layer $i$ is normally distributed along the $z$ axis around the layer center $\mu_{i}$ with variance $\sigma_{i}^{2}$, $\varphi$ represents the coarse-graining function defined in Eq. (1), and $N$ the number of particles within a coarse-graining length scale of each point along the profile. By fitting each layer's variance $\sigma^{2}$ to best agree with the measured density profile, values for each layer's mean squared displacement $\left\langle\delta z^{2}\right\rangle$ were obtained for all smooth base experiments. Only smooth-base experiments were used for this analysis because the mean particle velocity is parallel to the base, which is not true for the top moving layer of the rough-base experiments. Besides, the rough-base experiments include different depths of a bottom static layer, and we wish to compare the vertical displacement of layers of particles in motion. Interestingly, we find that all experiments show a similar seemingly linear increase in $\delta z$ with height, as evidenced by Fig. 7 .

The displacements obtained for the bottom layer match the particle tracking error, implying the vertical fluctuation in this bottom layer is minimal. In fact, direct observation of the experimental videos shows that disks in this layer do not fluctuate around the layer center at all, but mostly slide over the base. The mean vertical displacement from the layer 


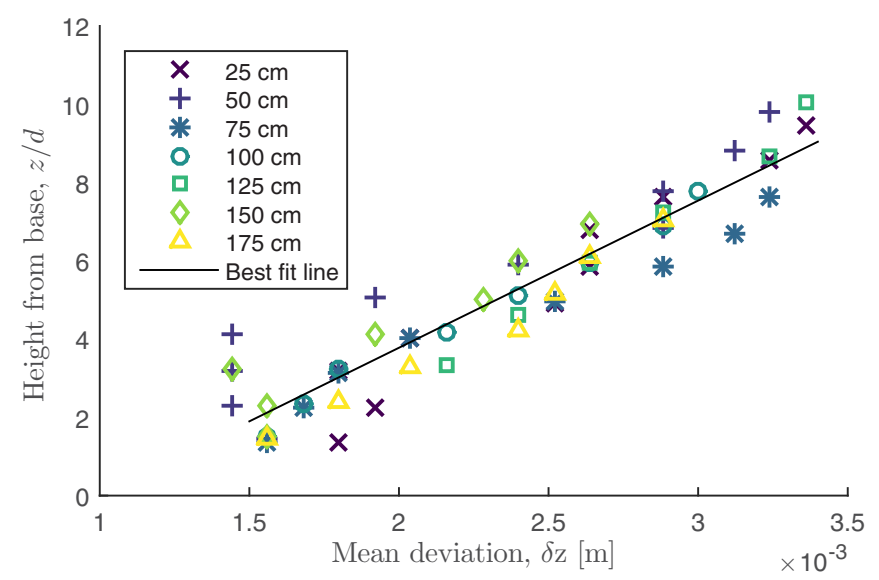

FIG. 7. Fitted standard deviation of the mass normal distribution around each layer center, representative of the mean particle deviation from the layer center, plotted against the layer height. Results are shown for the seven experiments carried out over a smooth base.

center of these disks is measured to be of $1 \mathrm{~mm}$, which coincides with the difference in radius between the smallest ( $r=$ $5.5 \mathrm{~mm})$ and largest $(r=6.5 \mathrm{~mm})$ disk. Therefore, the mean displacement obtained for the bottom layer is reasonable.

\section{B. Velocity profiles}

We apply the expression for coarse-grained velocity presented by Weinhart et al. [43] with $\varphi$ as defined in Eq. (1) and $w_{z}=3 d$ and $w_{x}=5 d$. Figure 8 shows the resulting plots of downstream velocity against height from the base for experiments carried out over both smooth and rough bases, and measured at seven different positions downstream.

The velocity profiles seem to evolve downstream, particularly in the rough-base case, where the flow thins visibly. However, Fig. 9 shows that by plotting the velocity against depth from the free surface rather than against height from the base, the velocity profiles in fact collapse. Moreover, the flowing layer of the rough-base experiments has the same thickness as the flow over a smooth base.

The velocity profiles reflect that while the particle density remains constant and uniform throughout all experiments, the

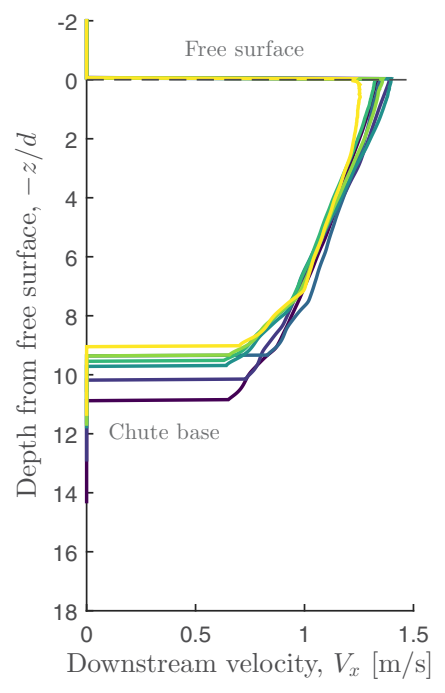

(a)

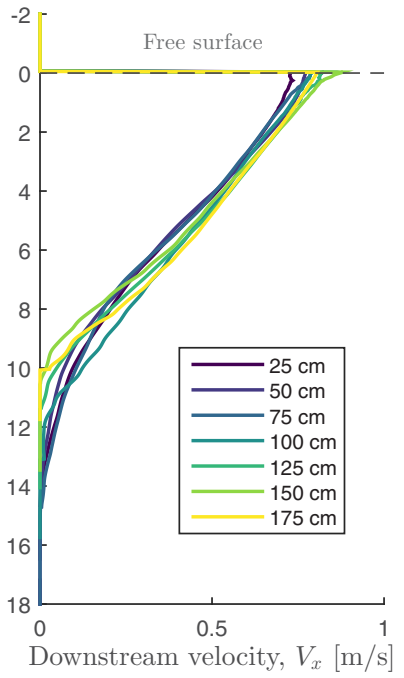

(b)
FIG. 9. Collapse of the coarse-grained downstream velocities profiles at seven positions down the chute for flows over two different topographies. Both figures use the same color scheme for the profiles measured at the different downstream positions.

flux depends strongly on the topography. In the smooth-base case there is high slippage and a quasilinear increase of the downstream velocity with height. Faug et al. [50] reported obtaining high slippage and a Bagnold profile in similar experiments over gentle slopes, but with only 10 layers of particles it is difficult to confirm or rule out such a result, as opposed to a linear profile. Except in the experiment recorded at a position closest to the hopper $(25 \mathrm{~cm})$, all profiles are remarkably similar to each other with no obvious thinning or acceleration. This implies that the gravitational downstream forces are balanced by the friction introduced by the base. If the chute were inclined less than the $20^{\circ}$ it was built at, the gravitational component would not be large enough to maintain a flow. In other words, the experiment is inclined at the dynamic angle of repose corresponding to a near monodisperse system of disks made of ClearFlex 50, flowing within the channel described in the previous chapter over a smooth base. In contrast, by forming a 2D pile of disks and tilting it slowly and smoothly until the first disks

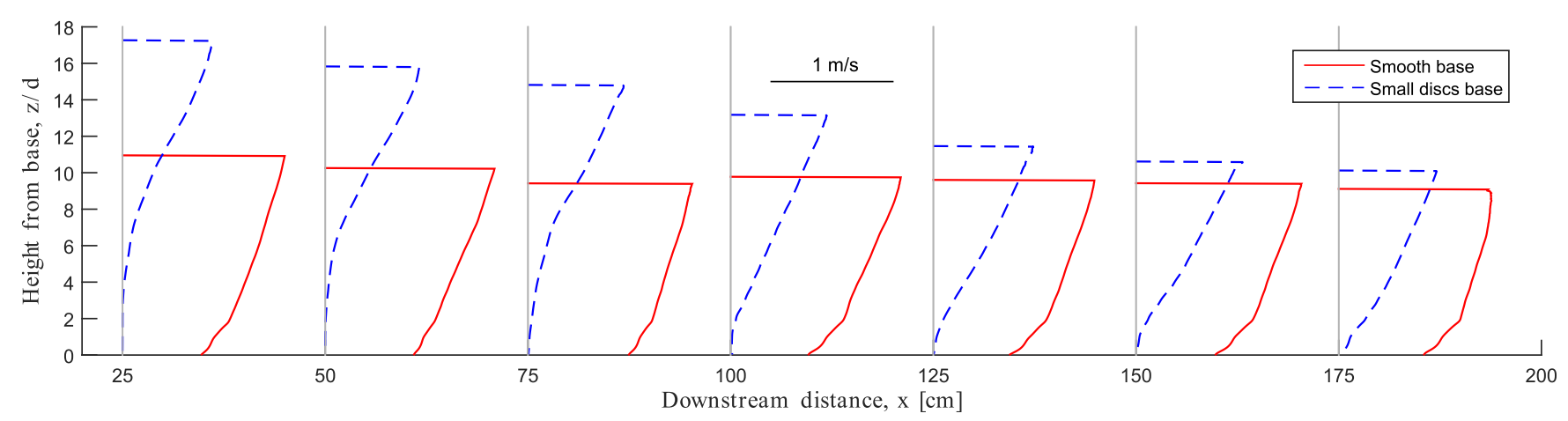

FIG. 8. Evolution of the coarse-grained downstream velocities profiles at seven positions down the chute for flows over two different topographies. 
topple, it was found that the system's static angle of repose is $31 \pm 2^{\circ}$, which is as expected larger than the dynamic counterpart.

The picture is completely different when basal roughness introduces shear into the system. Here, the dynamic angle of repose is larger because the increased basal friction requires a stronger gravitational downstream component to balance the higher shearing forces. A static layer therefore forms at the bottom, and it is this layer that thins while the moving layer on top maintains its thickness and quasilinear velocity profile throughout. The free-surface forms an angle of $25^{\circ}$ degrees to the floor, $5^{\circ}$ larger than in the smooth-base case. Furthermore, we verified that all particles in the flowing layer travel parallel to the free surface, confirming that the quasistatic layer effectively increases the system dynamic angle of repose. The static layer is referred to as a superstable heap (SSH), and many authors [51] have attributed it, and the shape of the velocity profile of the flow above it [52], to the effect of the chute side-wall friction on the flowing particles.

As the hopper depletes and the flux rate decreases, the inclination angle of the free surface decreases from $25^{\circ}$ when the flow is in steady state to $23^{\circ}$ when the flow stops completely. This is indicative of an increase of friction with depth, as particles need a steeper angle to flow when they are under pressure. This result suggests there is a relationship between the flowing layer thickness and the angle made by the free surface, as proposed by Taberlet et al. [51] assuming a Coulomb-type friction at the side walls. In any case, friction at the side walls has a significant effect on the flow velocity profile, but lack of photoelastic response in fast-moving rattlers (Fig. 5) regardless of particle depth, suggests side-wall friction is not large enough to interfere with the measurement of interparticle forces.

The observations presented so far agree with other reports $[10,53,54]$ that say that the packing fraction in slowintermediate flows is uniform, and is only a decreasing function of the inclination angle. In all experiments, a combination of relatively slow hopper discharge flux and small inclination angles causes the flow to have the minimum recorded flow thickness possible for gravity-driven flows [55]. The observations made here therefore correspond to flows with the slowest possible speed and thickness. It stands to reason that the mean packing fraction in these cases would tend to the $2 \mathrm{D}$ random close packing fraction.

Also confirming the slow and thin regime is the fact that the velocity profile is linear [17,56]. All flows (excluding the static layer in rough-base experiments) have approximately 10 diameters $d$ in thickness, which is significantly below the estimated thickness of $20 d$ at which Bagnoldian velocity profiles are observed [57]. However, in this case, the side-wall effect is also partially responsible for the velocity profile linearity [53].

No less significant is that the ability to compare the experiments described in this work with those reported by other groups confirms the small effect of the normal restitution coefficient $e$ on the flow behavior [58,59]. The restitution coefficient of Clear Flex 50 is lower than that of the particles used in said studies, yet the kinematics of these photoelastic flows are analogous to all flows in the same regime, regardless of the constitutive particle material.

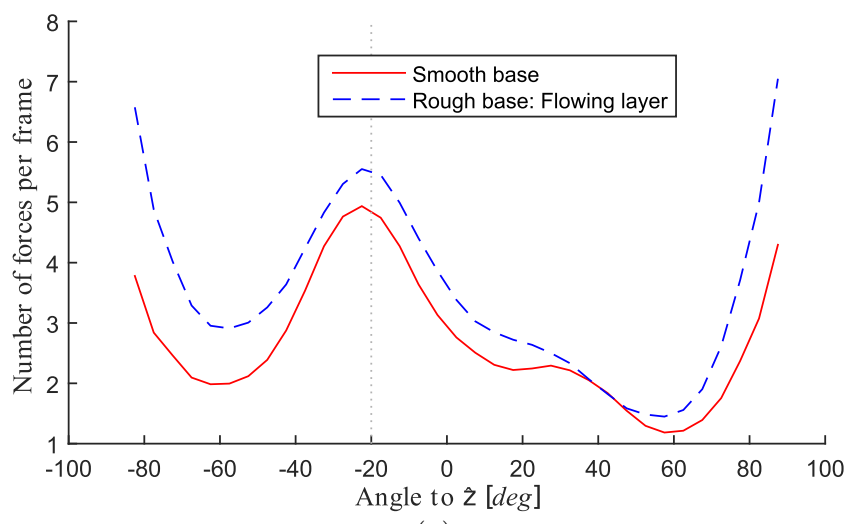

(a)

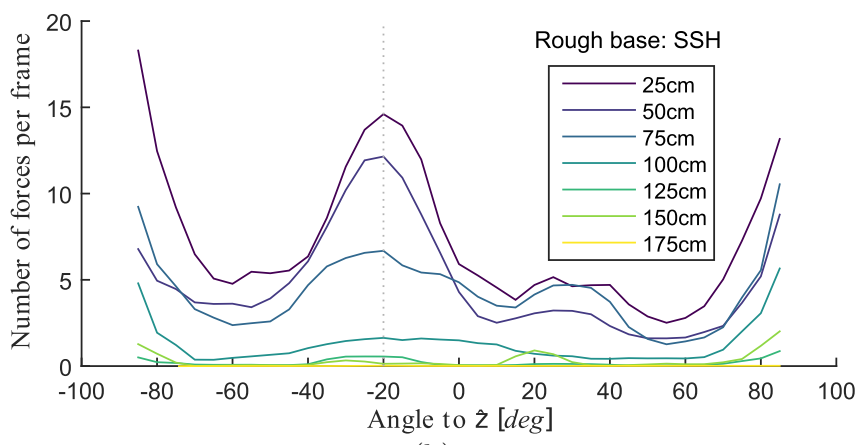

(b)

FIG. 10. Average number of forces per frame measured acting in angles to the cross-flow direction $\hat{z}$.

\section{RESULTS}

\section{A. Forces statistical analysis}

\section{Force numbers, means, and directions}

By collecting all the information on the instantaneous interparticle force magnitudes, directions, and application points, we are able to extract a network of forces within the avalanching flow. The total number and mean magnitude of interparticle forces observed in each direction varies. By binning each force according to the direction they are applied in into $5^{\circ}$ wide bins between $-90^{\circ}$ and $90^{\circ}$, the preferred direction and the mean magnitude of the interparticle forces is revealed.

Figure 10 shows the mean number of forces per frame $N_{f}$ recorded within each bin for the experiments performed over both a smooth and rough base. The forces measured in the rough-base experiments were further separated according to whether they were measured at depths of up to $11 d$ from the free surface (corresponding to the flowing layer, according to Fig. 9), and those at $z>11 d$ (within the SSH). The flowing layers in both types of experiments have approximately the same thickness, which is consistent along the chute, so Fig. 10(a) shows the mean number of forces measured in each direction per frame in the flowing layers over both types of basal roughness, averaging the results obtained at all 7 positions along the chute. On the other hand, because the static layer decreases in thickness along the chute, we plot $N_{f}$ measured at each position along the chute in Fig. 10(b). In all cases, the data are time averaged over 500 frames per experiment. 


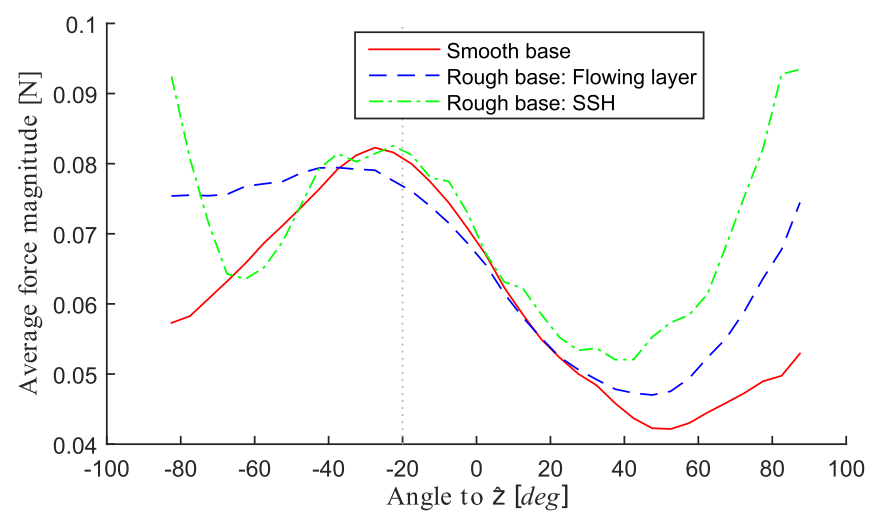

FIG. 11. Mean force magnitude measured acting in angles to the cross-flow direction $\hat{z}$.

From Fig. 10(a) we observe peaks in the number of forces measured to act at angles of $90^{\circ}$ (parallel to the chute base), and close to $-20^{\circ}$ (direction of gravity). At depths $z>11 d$ we observe the middle peaks coincide exactly with $-20^{\circ}$, as seen in Fig. 10(b), but as the static layer thins the number of forces measured decreases. In contrast, in the flowing layer the middle peak is slightly skewed to a more negative angle, which we attribute to the friction accompanying interactions between particles in different layers. In all cases, we see that the ratio of forces acting at $90^{\circ}$ to the number acting at $-20^{\circ}$ is larger in all rough-base experiments than in those with smooth base.

Figure 11 plots the mean force magnitudes $\langle F\rangle$ in each direction, for the flowing layers in the smooth- and rough-base experiments and within the static layer in the latter case. In all three cases we measure peaks in mean force magnitude around the direction of gravity. In the smooth-base case we observe that the forces acting at $90^{\circ}$ are significantly weaker than those at $-20^{\circ}$. In contrast, in experiments over a rough base the forces at $90^{\circ}$ are as important in the flowing layer, and even more significant within the $\mathrm{SSH}$, than those at $-20^{\circ}$. In addition, we notice that in rough-base experiments, peaks in $\langle F\rangle$ are more pronounced in the static layer than in the moving layer.

From Figs. 10 and 11 we conclude that force chains tend to form preferentially in the directions of the two external forces acting on the flow bulk: gravity and basal friction. When particles in different layers interact, if they move at different speeds, friction causes the force chains acting against gravity to skew slightly to slightly more up-slope values. Moreover, we measure less and weaker force chains acting parallel to the chute base in smooth- than in rough-base experiments. We associate this observation with the high slippage at the base [Fig. 9(a)] and weaker friction between layers within the flow. On the other hand, in the rough-base case we observe more and stronger force chains forming in the direction of friction acting on the particle layers. We observe that the peaks are sharper in the static layer, suggesting force chains bend and branch more in the moving layer. From the fact that we do not observe stronger forces at $-20^{\circ}$ in the $\mathrm{SSH}$ than in the moving layer, we deduce that particles in force chains carry similar loads. The extra weight supported by the SSH is spread into more force chains, that are not necessarily stronger.

\section{Force-chain fluctuations}

The downstream velocity profiles for the rough base, which were presented in Fig. 8, suggest a static layer forms at the channel base to effectively increase the flowing layer angle of inclination to its new dynamic angle of repose. The flux of disks, measured as $Q=\int \mathbf{v} \cdot d \mathbf{A}$, is confirmed to be constant for the different experiments recorded at the various points of its development.

Separately, the occurrence of high intensity pixels in the experimental images are indicative of the presence of a force chain. These chains mostly span from the channel base until the second or third layer from the top, independently of velocity. From direct observation we see that the density of force chains and the duration or persistence of an individual chain decreases with the distance to the free surface.

Pouliquen and Forterre (2009) [11] used a similar intuitive assumption as the basis of an original attempt to model the nonlocal rheology of sheared granular materials. They formulated that force-chain rearrangements are self-activated, meaning that they are a consequence of rearrangements elsewhere in the system. In their analogy with viscous liquid state transitions, it is the rate of rearrangements in the force network that plays the role of temperature in thermal systems, rather than individual particle fluctuations. They then assumed the rate of plastic deformation proportionally affects the rate of generation of new random force network within a granular flow.

Since in our experiments the photoelastic response is indicative of force-chain presence, we can measure the duration for which each point in the image forms part of a force chain. We can thus offer the first experimental validation of the assumption that shear rate $\dot{\gamma}$ is proportional to force-chain fluctuation. We obtain shear rate profiles from the velocity profiles (Fig. 8) and compare them to the fluctuations in pixel intensity at each point in the image. We define, through first-order finite differences, the mean rate of change of the intensity $I$ at a pixel with coordinates $x$ and $y$ in the experimental images as

$$
\overline{\delta I}(x, y)^{2}=\frac{1}{N} \sum_{t=1}^{N}[I(x, y, t+1)-I(x, y, t)]^{2},
$$

where the total number of frames $N=500$ and $t$ is the extracted frame number. To account for the higher density of force chains at lower depths, we normalize by dividing this result by the mean intensity $\bar{I}(x, y)$. We calculate $\overline{\delta I}(x, y)^{2}$ by averaging over all pixels at equal depths, and then dividing the result by the sum of the mean intensities squared for that row:

$$
\left\langle\delta I(y)^{2}\right\rangle=\frac{\left\langle\overline{\delta I}(x, y)^{2}\right\rangle_{x}}{\left\langle\bar{I}(x, y)^{2}\right\rangle_{x}} .
$$

Figure 12 plots in thick black lines the velocity profile coarse grained at each downstream position. The solid red lines represent the shear rate calculated by first-order finite differences from the velocity profiles. Finally, the dasheddotted blue lines plot the normalized squared pixel intensity fluctuation $\delta I^{2}$ (measured in arbitrary units). Results for both $\dot{\gamma}$ and $\delta I^{2}$ are smoothed using the MATLAB function smooth with factor 5. This analysis was performed only for 

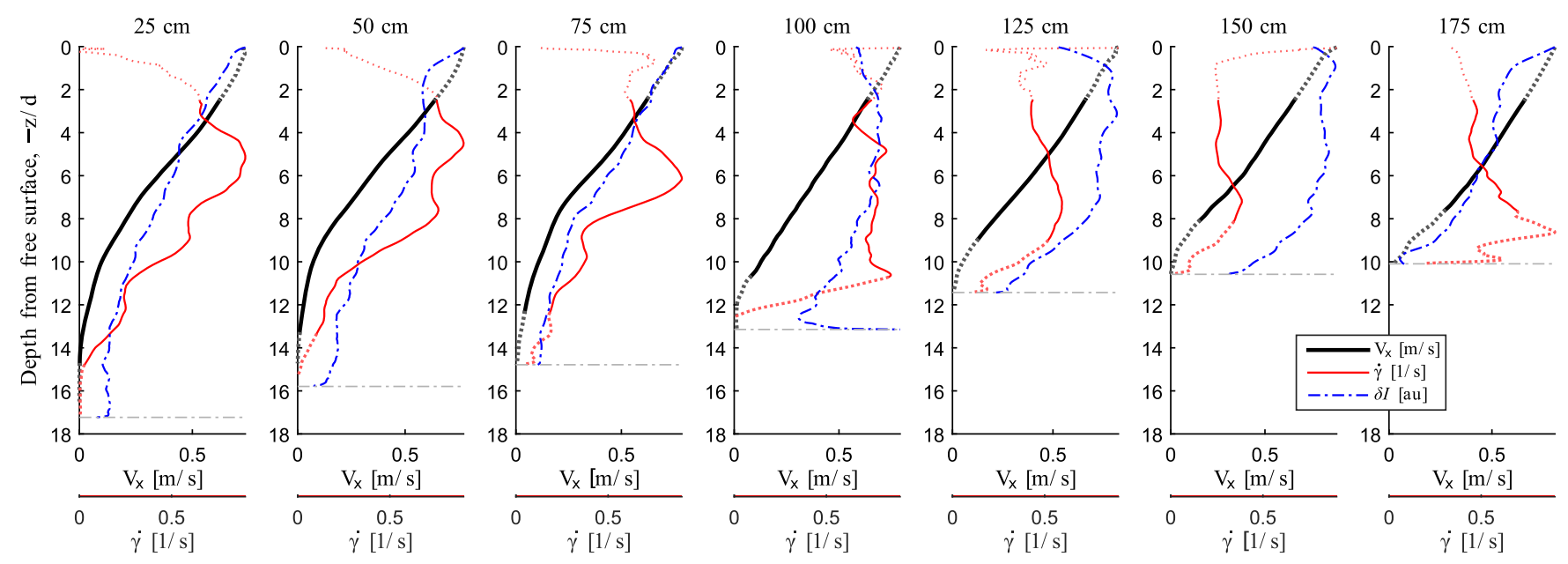

FIG. 12. Comparison between the force-chain squared fluctuations [Eq. (3)], mean downstream particle velocity, and shear rate profiles for experiments performed over a rough topography, measured at seven different downstream positions. The velocity profiles correspond to the same measurements shown in Figs. 8 and 9, and the shear rates were calculated from them by first-order finite differences.

experiments carried out over a rough base as they exhibit a wider range of velocities than the smooth base experiments, and contain the particularly interesting regions where particles are stationary. Each of the seven plots shows information obtained at different distances from the beginning of the chute.

Figure 13 plots the measured values for the fluctuations $\delta I$ against the corresponding shear rate at the same point in the flowing layer (that is, depth $z<10 d$ ). Data within distances of $w_{z}$ from the base and from the free surface are ignored because the velocity measurements in this region are affected by the coarse-graining process.

Figure 13 shows noisy data that at first glance could suggest there is indeed a monotonic relationship between the average downstream shear rate and the definition of

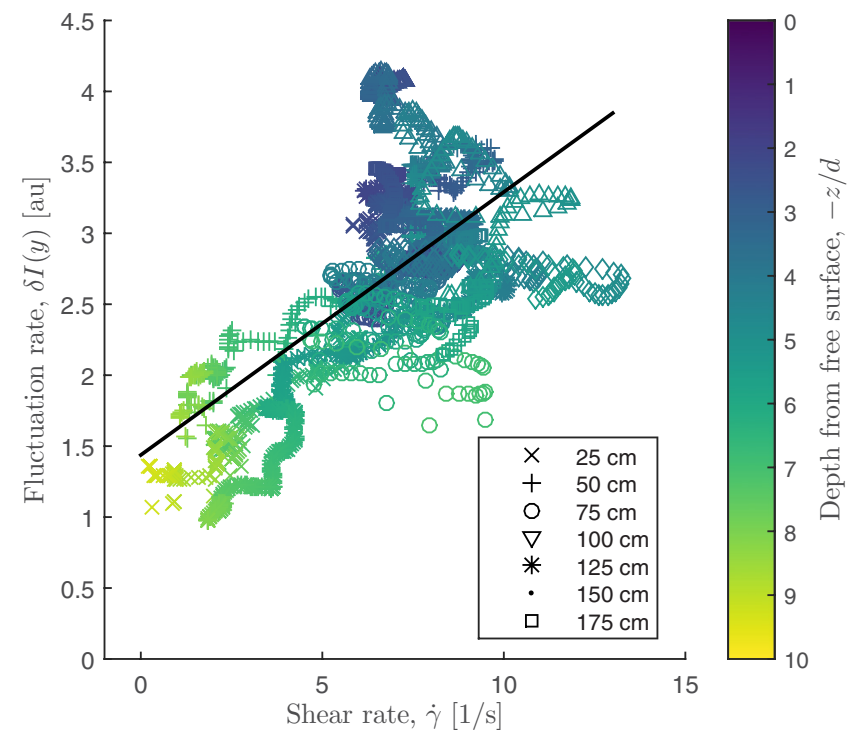

FIG. 13. Pixel intensity fluctuation rate $\delta I(y)^{2}$ plotted against the downstream velocity measured at the respective $y$ coordinate. The data color (or shade of gray) represents the depth to which that data point corresponds. fluctuation $\delta I$ used here [Eq. (3)]. The correlation coefficient between the two measured variables is 0.64 , which is too low to state with certainty that the relationship is linear. However, a validated monotonic relationship between $\dot{\gamma}$ and $\delta I$ confirms that a rearrangement of particles affects the force network a non-negligible distance away. This has important implications for rheology as it is the principle on which the latest nonlocal models are based $[11,12,60]$.

On the other hand, we observe from Fig. 12 that the force chains fluctuate where particle velocities, and shear rates, are zero in the static layer. It has been previously suggested that there exists an ensemble of force network arrangements for a given fixed packing configuration [9,61,62], and our results imply that particle rearrangements in the flowing layer triggers rearrangements of the force network in the static layer.

\section{B. Stress tensor component profiles}

Considering a fully developed, steady flow that does not change in time nor along the downstream direction $x$, momentum balance requires

$$
-\nabla \sigma+\rho \mathbf{g}=0
$$

where $\sigma$ represents the flowing system stress tensor, $\rho$ the density, and $g$ the acceleration of gravity. Assuming constant and uniform density $\rho$, resolving the streamwise $(x)$ and crossflow $(z)$ directions and solving for $\sigma$ predicts a hydrostatic increase in pressure and shear,

$$
\begin{aligned}
& \sigma_{z z}=(h-z) \rho g \cos \theta_{f s}, \\
& \sigma_{z x}=(h-z) \rho g \sin \theta_{f s},
\end{aligned}
$$

where $h$ represents the height of the free surface over the chute base and $\theta_{f s}$ the angle made by the free surface in the laboratory frame of reference.

The consistency in time and $x$ direction of the density and velocity profiles over a smooth base implies this is indeed a steady, fully developed flow. The packing fraction profiles (Fig. 6) suggest the density is indeed constant and 
uniform, and we furthermore assume that even if the flow were compressible, a change in solid volume fraction makes very little difference to the linear pressure distribution (Fig. 4 in Barker et al. [63]). Therefore, we expect the corresponding pressure and shear profiles to behave as predicted by Eq. (6). To compare that model to the discrete forces measured experimentally, the coarse-graining equations originally put forward by Goldhirsch [42] and later extended by Weinhart et al. [43] were applied to obtain continuous expressions for the four stress tensor components. The same coarse-graining function, Eq. (1), was used for this purpose, with $w_{z}=3 d$ and $w_{x}=5 d$.

We calculated the stress tensor component profiles according to Weinhart et al. [43] for each frame and then averaged over the 500 frames extracted from each experiment. Because the magnitudes of $\sigma_{z z}$ and $\sigma_{z x}$ depend only on depth from the free surface and angle of inclination, which are constant along the chute, all seven profiles collected along different downstream positions collapse, for both smooth- and rough-base experiments. In Fig. 14 we show the mean stress component profiles in solid lines, and bound the confidence intervals in dashed lines. The bounds of this interval are determined by the standard error of averaging the seven profiles, proving they all collapse within acceptable limits. Different stress tensor components are drawn in different colors (shades of gray), while the gray straight lines show the hydrostatic gradient predicted by Eq. (6) for $\rho=\rho_{\mathrm{CG}}$ and measuring $h$ to be the average height of a spline though the highest points of the disks on the flow top layer. Within a coarsegraining length $w_{z}=3 d$ from both the base and free surface, the lines are shown in faded colors, as the coarse-grained results here are likely to be affected by the closeness to the boundaries.

In the rough-base experiments, it is the static layer that thins with distance downstream (Fig. 9) in classic superstable heap (SSH) behavior [51]. From Fig. 8 we saw that the flowing layer remains practically constant in thickness and the velocity profile. It follows that the flowing layer should experience a hydrostatic pressure increase, but scaling with the cosine of the effective layer inclination angle $25^{\circ}$ rather than with the inclination of the chute $20^{\circ}$. Figure $14(\mathrm{~b})$ shows the components of the stress tensor resolved relative to the free surface and to the normal to that direction, having rotated the camera frame of reference by $5^{\circ}$ to match the new $\hat{x}$ to the direction of the free surface.

\section{Principal stresses}

Since we can obtain from experiments all four components of the 2D internal stress tensor $\overline{\bar{\sigma}}$, we can calculate the inplane principal stresses. These are defined as the two (for 2D systems) pressures $\sigma_{1}$ and $\sigma_{2}$ that acting in a what is known as the principal stress directions, have an analogous effect on the system, with no shearing stresses, than the full $\overline{\bar{\sigma}}$ in the original plane.

The magnitudes of the principal stresses are the eigenvalues of the stress tensor, and the corresponding eigenvectors represent the directions of the axes in the principal stress plane. The eigenvalues corresponding to the collapsed, depthdependent stress tensors presented in Fig. 14 are plotted against depth from free surface in Fig. 15.

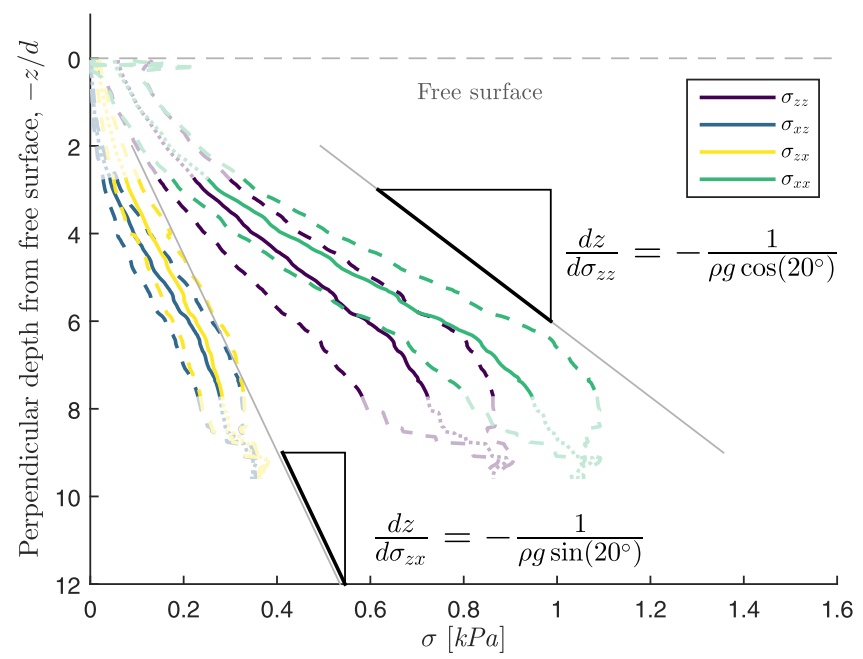

(a)

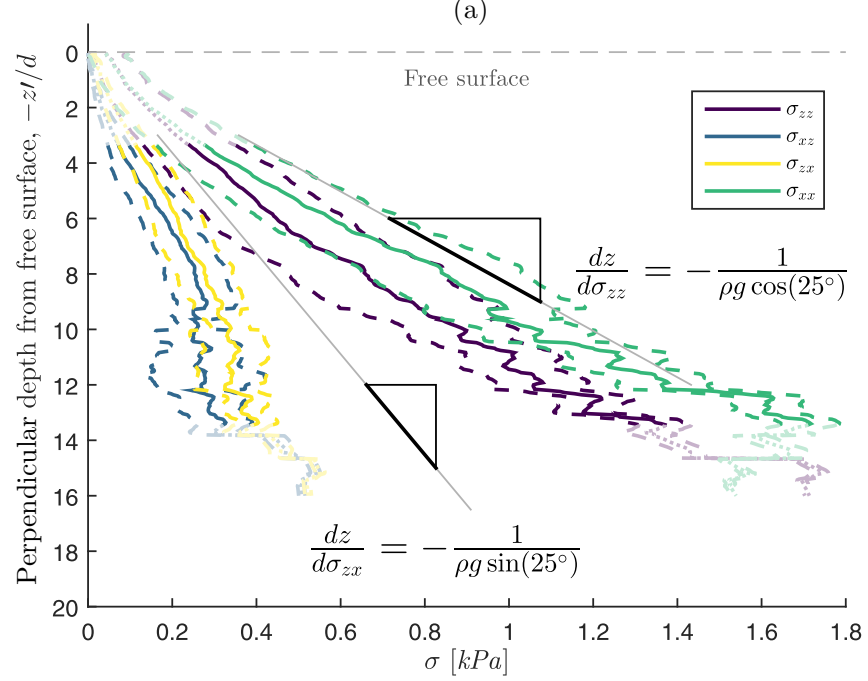

(b)

FIG. 14. Coarse-grained 2D stress tensor component profiles for (a) smooth-base and (b) rough-base experiments. The profiles resulting from averaging the seven experiments measured at different downstream locations are plotted in solid lines, while the dashed lines delimit the standard error of he averaging. The straight gray lines show the gradient corresponding to a hydrostatic increase in pressure as predicted by Eq. (6) with $\theta_{f s}=20^{\circ}$ for (a) and $\theta_{f s}=25^{\circ}$ for (b).

It was found that the corresponding eigenvectors for the coarse-grained stress tensor form an orthogonal basis. We therefore report the direction of only one principal stress, the principal orientation $\alpha_{p}$, bearing in mind that the second direction forms a normal angle to this one. To facilitate comparison, we calculated $\alpha_{p}$ for the coarse-grained stresses in the camera frame of reference, for experiments over both types of topography. The principal orientation corresponding to the principal stresses just obtained are also plotted against depth from the free surface in Fig. 16.

Interestingly, the principal stress magnitudes are very similar in both smooth- and rough-base experiments. On the other hand, the angle $\theta_{p}$ is lower for the smooth-base than for the rough-base experiments, but in both cases the principal orientation lies between the direction of gravity $\left(90^{\circ}\right.$ to the 


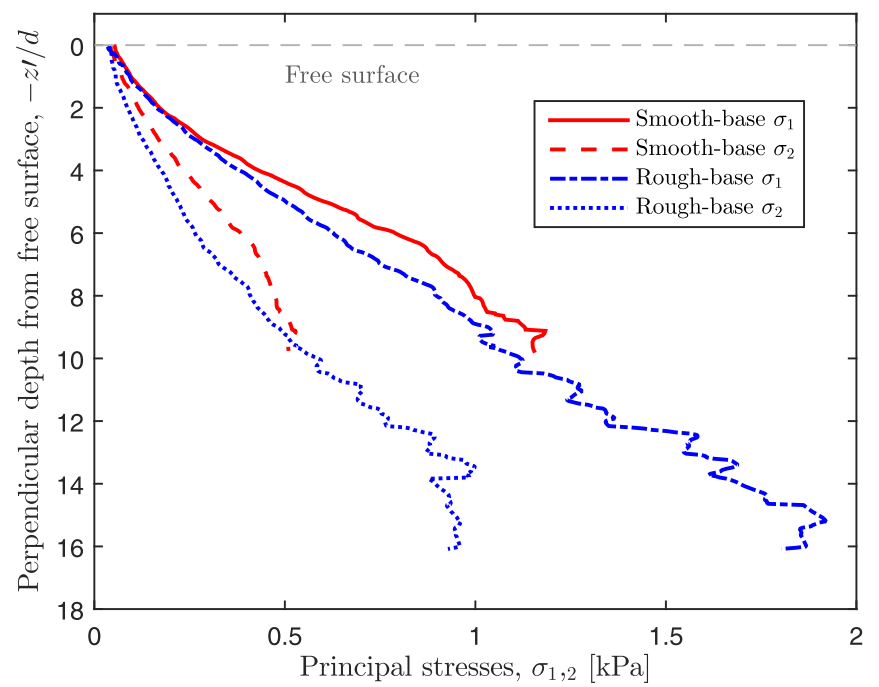

FIG. 15. Magnitudes of the principal stresses for the stress tensors obtained by collapsing the results of all experiments of equal type. The dashed blue lines represent the results of experiments performed over a smooth-base while solid red lines correspond to rough-base experiments.

cross-flow direction $\hat{z})$ and basal shear $\left(20^{\circ}\right.$ anticlockwise from $\hat{z}$, see Fig. 10). These are the two body forces acting on the flow bulk, so it seems reasonable that the main principal stress would be directed somewhere in-between them $\left(35^{\circ}-50^{\circ}\right.$ anticlockwise from $\left.\hat{z}\right)$.

We propose that the principal orientation in these experiments depends on the local relative importance between interlayer shear and hydrostatic pressure. In the smooth-base case, there is high slippage at the chute base and the shear rate is smaller than in the rough-base case. Hence, shearing forces are less important in the smooth-base case. On the other hand, hydrostatic pressure depends only on depth from

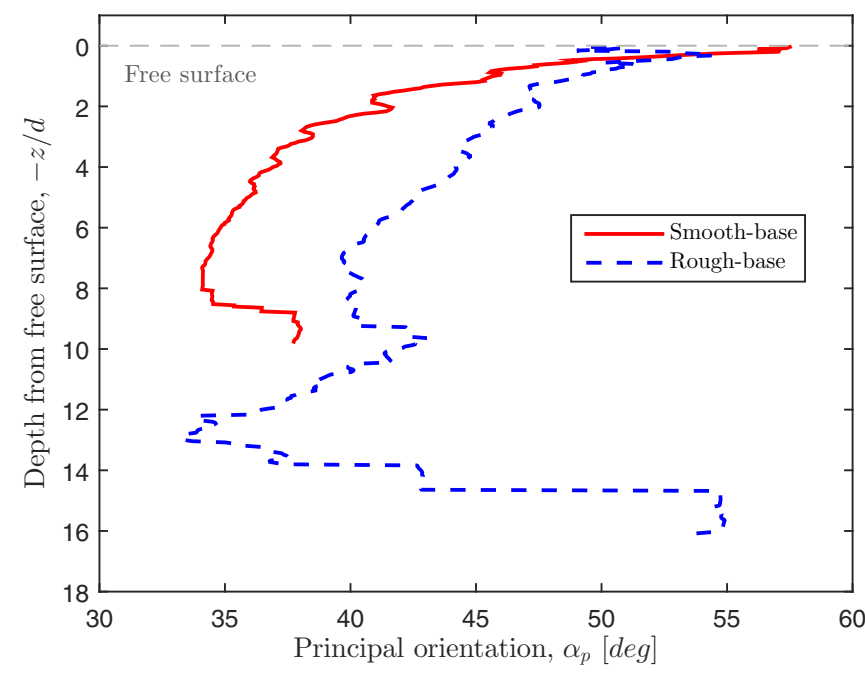

FIG. 16. Orientation of the system principal stresses and their dependence with depth. The dotted and dashed-dotted blue lines represent the results of experiments performed over a smooth-base while solid and dashed red lines correspond to rough-base experiments. the free surface and inclination angle. Although the flowing layer in the rough-base case has the same depth of $\sim 10 d$ as the flow over a smooth base, the superstable heap ( $\mathrm{SSH}$ ) increases the effective angle of inclination of the flowing layer in the former case. Therefore, in the rough-base case shearing forces $\left(90^{\circ}\right.$ from $\left.\hat{z}\right)$ are more relevant than in the smooth-base case, and the hydrostatic pressure $\left(20^{\circ}\right.$ from $\left.\hat{z}\right)$ is less so. Thus, we infer from the larger $\theta_{p}$ in the top $10 \mathrm{~d}$ that the principal orientation leans toward the direction of shear in the rough-base case more than in the smooth-base case, due to the larger relative importance of shear in the system.

Nevertheless, both lines in Fig. 16 follow the same trend within the flowing layer (approximately the top $10 \mathrm{~d}$ in both cases). Close to the free surface the only surface force acting on the particles is shear (hydrostatic pressure is minimal), so the main principal stress tends toward $90^{\circ}$. The velocity profile within the flowing layers was determined to be linear, so interlayer shear remains constant in depth within these flows. As the hydrostatic component increases with depth, the principal orientation leans more toward gravity (which acts at $20^{\circ}$ to the cross-flow direction $\hat{z}$ ). Then, shear changes suddenly at the chute base (in the smooth-base case) as well as in the transition between the flowing and static layers (in the rough-base case), and so $\theta_{p}$ increases at these boundaries. Within the static layer that forms the SSH (only visible in the rough-base experiments) a dip in $\theta_{p}$ is observable as shear is most relevant at the boundary with the flowing layer ( $\sim 10 d$ depth) and with the chute base (most visible at $\sim 16 d$ depth).

\section{CONCLUSIONS}

The first main result of the work described in this paper is the successful ability of the photoelastic technique in quantifying forces in dynamic systems. This paper summarizes how the technique works and the steps taken to obtain force magnitudes from experimental images. In addition, we allude to different materials tested and open-source software other researchers could refer to if they wanted to apply the technique themselves. The key is to choose the photoelastic base material and particle dimensions such that the range of forces that the technique measures most accurately coincides with the force magnitudes expected within the experiment. On one hand, the lower limit of the technique sensitivity is determined by the material photoelastic coefficient and dimensions, as there must be a clearly visible photoelastic response for any force to be measured. On the other hand, the optimization problem solved in order to calculate forces is prone to fall into local minima rather than global minima when the forces are large enough to raise the fringe number over $N=1$. Hence, the force that would produce a fringe pattern of order 1 is the upper limit of the force range for which the technique is most reliable. Furthermore, the experimental error increases with the number of forces applied on each particle, so the technique is more appropriate for systems with low force-chain density.

We apply the photoelastic technique to obtain innovative experimental measurements of forces within the bulk of 2D free-surface, gravity-driven, dry granular flows. Side-wall effects play a significant role in the kinematics of the flows 
produced, but the intrinsic relationships between flow kinematics and dynamics discussed here are nonetheless applicable to all dry granular avalanches. In particular, by coarse graining the results of this original experiment, we test the extent to which such a discrete system can be modeled as a continuum.

From a discrete point of view, at most 30\% (although the exact proportion depends on depth) of the flow constitutive particles carry a significant load. These particles, that form part of force chains, transport loads one or two orders of magnitude larger than a single particle weight. Moreover, we determined that, within the dense flow, force chains rearrange at a rate that follows a monotonic relationship with the local shear rate. However, this relationship ceases to hold for low to null velocities. Regardless of the force network dynamics, we have shown through coarse graining that the average stress tensor is equivalent to that of a continuous flow, including a hydrostatic increase of pressure with depth (Fig. 14).

Furthermore, we determined that force chains form preferentially in the directions of the forces acting on the bulk. In this case, the two external force sources are gravity and the shear induced by the basal topography, and a larger number of force-chain forces are directed in these two directions (Fig. 10). From comparisons between the results from configurations where gravity and basal shear bear different relative importance, we propose that the system main principal stress is directed somewhere in-between the two, leaning closer to the the most relevant locally.

\section{ACKNOWLEDGMENTS}

We are very grateful for the many enlightening contributions made by the members of the Daniels Lab at North Carolina State University, USA, including K. Daniels, M. Shearer, J. Kollmer, and Z. Tang. We also appreciate our extremely useful discussions with N. Gray about superstable heaps. Finally, we thank technicians D. Page-Croft, P. Mitton, C. Hitch, A. Denson, and J. Milton for their invaluable help and technical support in the construction and maintenance of the experimental apparatus. N.M.V. is supported by a Royal Society Dorothy Hodgkin Research Fellowship Grant No. DH120121 and Royal Society Grant No. RG130403, A.L.T. is funded by a Royal Society Research Grant No. RG 73329 and the Cambridge Trust.

\section{APPENDIX: FORCE MEASUREMENT ERROR}

In order to investigate the measurement error specific to our experimental system, we numerically produced images of photoelastic fringe patterns for disks under $z=2,3$, and 4 concentric forces of equal magnitudes $F$, evenly distributed around the disk surface. These images were then reduced to a resolution similar to that in which the experimental disks are observed, and a random noise of the order of the experimental one was added to the figures. Then, the force magnitudes in the result were resolved by PEGS and plotted against the original $F$, as shown in Fig. 17.

From Fig. 17 we infer that there is a systematic error of approximately $0.05 \mathrm{~N}$ for forces below $0.5 \mathrm{~N}$ that originates

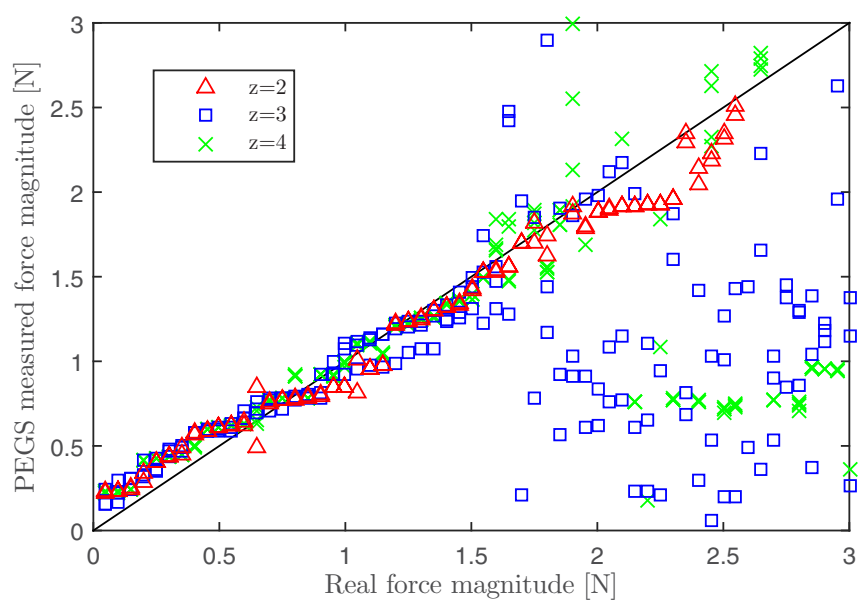

FIG. 17. Correlation between the real forces exerted on a photoelastic disk and the measured magnitudes. This allows us to estimate the technique force measurement error, dependent on the number of forces acting on a disk $z$.

from the experimental noise. The minimum force that can be consistently resolved by PEGS is $0.02 \mathrm{~N}$, which produces the minimum photoelastic response larger than the noise in the inner $95 \%$ of the disk. The edge is ignored because in the experimental images it often shows a marked intensity difference due to the edges of the disks being inevitably slightly rounded.

When the disk displays a large fringe number, the optimization algorithm is prone to falling into local minima rather than the global minimum. Hence, there is a maximum force magnitude beyond which the technique becomes unreliable, which depends on how many forces act on the disk. The limits are approximately $2.5 \mathrm{~N}$ when $z=2,1.6 \mathrm{~N}$ when $z=3$, and $1.2 \mathrm{~N}$ when $z=4$. However, the forces involved in the experiments are rarely stronger than $0.5 \mathrm{~N}$, and particles are rarely subject to more than 2 or 3 contacts that transmit such large loads. Below forces of $0.5 \mathrm{~N}$ the systematic error measured is similar for whatever $z$, so this value is subtracted from all measurements.

To summarize, the sensitivity lower bound of our photoelastic measurements was estimated at $0.02 \mathrm{~N}$ and depends on the inherent photoelastic response of Clear Flex 50, the thickness of the disks, and the image resolution intrinsic to the camera. The measurement of even smaller forces could be achieved by increasing either the disk thickness, the image resolution, or the material photoelastic coefficient, but in our current setup we are limited by the sensitivity lower bound of $0.02 \mathrm{~N}$. Then, a systematic error of $0.05 \mathrm{~N}$ exists for forces of magnitudes smaller than $0.5 \mathrm{~N}$. On the other hand, the random error of the technique can be as large as $20 \%$ between this lower bound and a critical value that depends on the number of forces acting on the disk. The reliability of the force measurements drops significantly when the loads on the disks exceed $2.5 \mathrm{~N}$ when $z=2,1.6 \mathrm{~N}$ when $z=3$, and $1.2 \mathrm{~N}$ when $z=4$. In our experiments, approximately 1 in 100 disks is subject to three or four force-bearing contacts, and in these cases two of them are usually dominant by at least an order of magnitude. 
[1] H. M. Jaeger and S. R. Nagel, Science 255, 1523 (1992).

[2] J. M. N. T. Gray, Annu. Rev. Fluid Mech. 50, 407 (2018).

[3] H. M. Jaeger, S. R. Nagel, and R. P. Behringer, Phys. Today 49(4), 32 (1996).

[4] T. S. Majmudar and R. P. Behringer, Nature (London) 435, 1079 (2005).

[5] C. S. O'Hern, S. A. Langer, A. J. Liu, and S. R. Nagel, Phys. Rev. Lett. 88, 075507 (2002).

[6] J. Brujić, S. F. Edwards, D. V. Grinev, I. Hopkinson, D. Brujić, and H. A. Makse, Faraday Discuss. 123, 207 (2003).

[7] E. I. Corwin, H. M. Jaeger, and S. R. Nagel, Nature (London) 435, 1075 (2005).

[8] D. S. Bassett, E. T. Owens, K. E. Daniels, and M. A. Porter, Phys. Rev. E 86, 041306 (2012).

[9] J. H. Snoeijer, T. J. H. Vlugt, M. van Hecke, and W. van Saarloos, Phys. Rev. Lett. 92, 054302 (2004).

[10] L. E. Silbert, D. Ertaş, G. S. Grest, T. C. Halsey, D. Levine, and S. J. Plimpton, Phys. Rev. E 64, 051302 (2001).

[11] O. Pouliquen and Y. Forterre, Philos. Trans. R. Soc. London, Ser. A: Math., Phys. Eng. Sci. 367, 5091 (2009).

[12] K. Kamrin and G. Koval, Phys. Rev. Lett. 108, 178301 (2012).

[13] T. Weinhart, A. R. Thornton, S. Luding, and O. Bokhove, Granular Matter 14, 531 (2012).

[14] D. M. Mueth, H. M. Jaeger, and S. R. Nagel, Phys. Rev. E 57, 3164 (1998).

[15] L. Bocquet, W. Losert, D. Schalk, T. C. Lubensky, and J. P. Gollub, Phys. Rev. E 65, 011307 (2001).

[16] E. Longhi, N. Easwar, and N. Menon, Phys. Rev. Lett. 89, 045501 (2002).

[17] G. Berton, R. Delannay, P. Richard, N. Taberlet, and A. Valance, Phys. Rev. E 68, 051303 (2003).

[18] T. S. Majmudar, M. Sperl, S. Luding, and R. P. Behringer, Phys. Rev. Lett. 98, 058001 (2007).

[19] J. Zhang, T. S. Majmudar, M. Sperl, and R. P. Behringer, Soft Matter 6, 2982 (2010).

[20] J. Tang and R. P. Behringer, Chaos 21, 041107 (2011).

[21] J. G. Puckett and K. E. Daniels, Phys. Rev. Lett. 110, 058001 (2013).

[22] K. E. Daniels and N. W. Hayman, J. Geophys. Res.: Solid Earth 113, B11411 (2008).

[23] E. T. Owens and K. E. Daniels, Europhys. Lett. 94, 54005 (2011).

[24] J. Estep and J. Dufek, J. Geophys. Res.: Earth Surf. 117, F01028 (2012).

[25] A. H. Clark, A. J. Petersen, L. Kondic, and R. P. Behringer, Phys. Rev. Lett. 114, 144502 (2015).

[26] J. Zhou, S. Long, Q. Wang, and A. D. Dinsmore, Science 312, 1631 (2006).

[27] M. Saadatfar, A. P. Sheppard, T. J. Senden, and A. J. Kabla, J. Mech. Phys. Solids 60, 55 (2012).

[28] R. Hurley, E. Marteau, G. Ravichandran, and J. E. Andrade, J. Mech. Phys. Solids 63, 154 (2014).

[29] E. G. Coker and L. N. G. Filon, A Treatise on Photoelasticity (Cambridge University Press, Cambridge, 1931).

[30] M. M. Frocht, Photoelasticity (Wiley, New York, 1941), Vol. 1.

[31] M. M. Frocht, Photoelasticity (Wiley, New York, 1948), Vol. 2.

[32] J. E. Daniels, K. E. nd Kollmer and J. G. Puckett, Rev. Sci. Instrum. 88, 051808 (2017).
[33] J. E. Kollmer, Photoelastic grain solver, https://github.com/ jekollmer/pegs.

[34] J. G. Puckett, State variables in granular materials: An investigation of volume and stress fluctuations, Ph.D. thesis, NCSU, 2012.

[35] J. Barés, D. Wang, D. Wang, T. Bertrand, C. S. O'Hern, and R. P. Behringer, Phys. Rev. E 96, 052902 (2017).

[36] J. Barés, Photoelastic methods, https://git-xen.lmgc. univ-montp2.fr/photoelasticity/main/wikis/home.

[37] K. E. Daniels, J. E. Coppock, and R. P. Behringer, Chaos 14, S4 (2004).

[38] J. Krim and R. P. Behringer, Pure Appl. Geophys. 168, 2259 (2011).

[39] A. H. Clark, L. Kondic, and R. P. Behringer, Phys. Rev. Lett. 109, 238302 (2012).

[40] N. Iikawa, M. M. Bandi, and H. Katsuragi, J. Phys. Soc. Jpn. 84, 094401 (2015).

[41] Y. Zhou, P. Ruyer, and P. Aussillous, Phys. Rev. E 92, 062204 (2015).

[42] I. Goldhirsch, Granular Matter 12, 239 (2010).

[43] T. Weinhart, A. R. Thornton, S. Luding, and O. Bokhove, Granular Matter 14, 289 (2012).

[44] D. R. Tunuguntla, Polydisperse granular flows over inclined channels, Ph.D. thesis, University of Twente, 2015.

[45] J. Zhang, R. P. Behringer, and I. Goldhirsch, Prog. Theor. Phys. Suppl. 184, 16 (2010).

[46] A. H. Clark, P. Mort, and R. P. Behringer, Granular Matter 14, 283 (2012).

[47] C. Goldenberg, A. P. F. Atman, P. Claudin, G. Combe, and I. Goldhirsch, Phys. Rev. Lett. 96, 168001 (2006).

[48] S. Meyer, C. Song, Y. Jin, K. Wang, and H. A. Makse, Physica A (Amsterdam) 389, 5137 (2010).

[49] T. Weinhart, R. Hartkamp, A. R. Thornton, and S. Luding, Phys. Fluids 25, 070605 (2013).

[50] T. Faug, P. Childs, E. Wyburn, and I. Einav, Phys. Fluids 27, 073304 (2015).

[51] N. Taberlet, P. Richard, A. Valance, W. Losert, J. M. Pasini, J. T. Jenkins, and R. Delannay, Phys. Rev. Lett. 91, 264301 (2003).

[52] P. Jop, Y. Forterre, and O. Pouliquen, J. Fluid Mech. 541, 167 (2005).

[53] G. D. R. MiDi, Eur. Phys. J. E 14, 341 (2004).

[54] N. Mitarai and H. Nakanishi, Phys. Rev. Lett. 94, 128001 (2005).

[55] O. Pouliquen and N. Renaut, J. Phys. II 6, 923 (1996).

[56] L. E. Silbert, J. W. Landry, and G. S. Grest, Phys. Fluids 15, 1 (2003).

[57] W. Bi, R. Delannay, P. Richard, and A. Valance, Phys. Fluids 18, 123302 (2006).

[58] J. Rajchenbach, Eur. Phys. J. E: Soft Matter Biol. Phys. 14, 367 (2004).

[59] J. T. Jenkins, Phys. Fluids 18, 103307 (2006).

[60] M. Bouzid, A. Izzet, M. Trulsson, E. Clément, P. Claudin, and B. Andreotti, Eur. Phys. J. E 38, 125 (2015).

[61] J. E. Kollmer and K. E. Daniels, EPJ Web of Conferences (EDP Sciences, Les Ulis, 2017), Vol. 140, p. 02024.

[62] J. E. Kollmer and K. E. Daniels, Soft Matter (to be published).

[63] T. Barker, D. Schaeffer, M. Shearer, and J. Gray, Proc. R. Soc. A: Math., Phys. Eng. Sci. 473, 20160846 (2017). 